\section{Glycolysis, Hydrolysis and Phosphorolysis in Corynebacterium diphtheriæ}

THE two culiurally distinct types of Corynebacterium diphtherioe-gravis and mitis-differ biochemically in that only the former glycolyses glycogen although both glycolyse simpler carbohydrates such as glucose and maltose. On examining the hydrolysing capacity of these bacterial types in vitro we found that both gravis and mitis yield cellfree extracts or autolysates which convert glycogen into fermentable reducing sugar. In view of the differential desmolytic behaviour of the living cells towards glycogen it is necessary to conclude that in glycolysis the glycogenase of living cells does not necessarily come into play. It seems probable rather that gravis achieves the degradation of polysaccharide into lactic acid through a reaction path in which hydrolysis takes no part.

The only difference in enzymic outfit between gravis and mitis in vitro so far observed by us, is that whereas extracts of gravis contain an active phos phatase, this enzyme is absent in extracts of mitis. It seems natural to connect the difference in glycolytic behaviour with the noted difference in the phosphorylation-dephosphorylation apparatus. Our findings support the theory ${ }^{1}$ that the desmolytic degradation of complex carbohydrates in vivo by muscle, yeast and bacteria may proceed not through hydrolysis but by a direct attack on the saccharide molecule through phosphorolysis $\left(^{2-5}\right)$.

A detailed account of this investigation will be given elsewhere.

Department of Hygiene

and Bacteriology,

Hebrew University, Jerusalem.

April 10.

${ }^{1}$ Leibowitz and Hestrin, Enzymolog., 6, 15 (1939); NATURE, 145, $671(1940)$; further papers in the press.

'Cori and Cori, Proc. Soc. Exp. Biol., 39, 337 (1938).

'Schaeffner, Naturwiss., 27, 195 (1939).

' Kiessling, Biochem. Z., 302, 50 (1939).

'Hanes, Nature, 145, 348 (1940) ; Proc. Roy. Soc., B, 128, 421 (1940).

\section{Position of the Carboxyl Group in Oleanolic and Related Acids}

Measurements recently carried out on monomolecular films of $\beta$ - and $\gamma$-oleananic acids and esters ${ }^{1}$ show that these compounds occupy areas very much smaller than those which are calculated on the basis of Haworth's triterpene formula (I), the limiting values for the two acids being 52 and 45 sq. A. respectively. In these two compounds there are no water-attracting groups, apart from the carboxyl group, which could be responsible for the effects observed, as in oleanolic acid itself ${ }^{2}$; the results suggest that the carboxyl group must occupy a position in one of the terminal rings. Of the possible formulations, the structure (II) for oleanolic acid satisfies the principal requirements.

The ready formation of lactones from oleanolic and related acids appeared at first to present a difficulty, but examination of models shows that lactonization should be possible with certain stereochemical
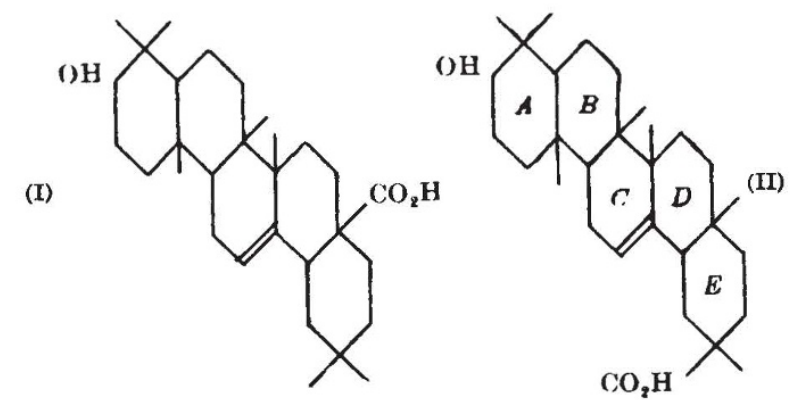

arrangements of rings $C, D$ and $E$, and a cis or a trans fusion of the last two rings. A cis fusion of rings $D$ and $E$ has already been suggested to account for certain reactions of quillaic acid ${ }^{3}$.

Philip B ILHam.

Laboratories of J. Lyons \& Co., Ltd., 149, Hammersmith Road, W.14.

George A. R. Kon.

Imperial College of Science and Technology, London, S.W.7.

1 Jacobs and Fleck, $J$. Biol. Chem., 96, 341 (1932).

Askew, J. Chem. Soc., 1585 (1936).

Bilham and Kon, J. Chem. Soc., 1469 (1940).

\section{Effect of Caffeine and Bromide on Dark Adaptation}

DURING the past two years we have been making an investigation of dark adaptation of the central region of the retina with special reference to the recovery occurring during the first minute after the end of light adaptation. The work has included some experiments on the effect of doses of potassium bromide and of caffeine on the subject. With doses of potassium bromide up to 25 grains no effect on the rate of adaptation or on the scatter of the observations was observed. With one subject who was used to drinking strong coffee and tea frequently no effect was produced with a dose of 8 grains of caffeine. With another subject whose consumption of tea and coffee may be regarded as at least average, a definite, though rather small, increase in the rate of adaptation was noted when doses of caffeine of up to ten grains were given. The increase was appreciable with 6 grains. In view of these results it is not surprising that at no time were we able to observe any effect due to fatigue or to minor ill-health.

It should perhaps be stated that the effect of a comparatively short period (17 days) of vitamin A starvation was considerably larger than any of the effects observed with the caffeine. The subject returned to nearly the original rate of adaptation as the result of a single large dose of vitamin A (24,000 I.U.).

These results in so far as they refer to fatigue are in agreement with those recently published by Semeonoff ${ }^{1}$. The rather small effects obtained with caffeine may be compared with experiments ${ }^{2}$ which appear to show that taking alcohol raises the vitamin A content of the blood considerably.

We wish to express our thanks to the Medical 